\title{
Tranexamic acid therapy in ulcerative colitis
}

\author{
D. Hollanders* \\ M.Sc., M.R.C.P.
}

\author{
JEAN M. THOMSON† \\ Ph.D.
}

\author{
PhILliP F. SCHOFIELD $\ddagger$ \\ M.D., F.R.C.S.
}

\begin{abstract}
Departments of *Medicine, $\nmid$ Haematology and $\ddagger$ Surgery, University Hospital of South Manchester, Nell Lane, West Didsbury, Manchester M20 8LR
\end{abstract}

\begin{abstract}
Summary
The antifibrinolytic drug tranexamic acid was assessed in controlling bleeding in 12 patients with left-sided proctocolitis. The study was designed as a double-blind cross-over trial employing a dose of $4.5 \mathrm{~g}$ of tranexamic acid/day together with identical placebo. A statistically significant reduction in rectal bleeding was achieved in $50 \%$ of cases with a minimum of side effects. Sigmoidoscopic grading of rectal mucosal appearances was improved. Frequency of diarrhoea and stool consistency were not altered.

Tranexamic acid may have a part to play in the management of selected patients with ulcerative colitis in whom bleeding is difficult to control with orthodox treatment.
\end{abstract}

\section{Introduction}

Ulcerative proctitis and procto-sigmoiditis are relatively common problems in medical practice, with current treatment being based mainly on the use of sulphasalazine and corticosteroid enemas. Despite this, persistent or recurrent rectal bleeding may remain a problem with both blood loss and malabsorption of iron resulting in anaemia in a large proportion of cases (Ormerod, 1968). Haemorrhagic stools also generate anxiety on the part of the sufferer who might otherwise be prepared to tolerate moderate diarrhoea. Any therapy which effectively controlled bleeding would therefore represent a definite advance in the management of this condition.

Although the mechanism of the mucosal haemorrhage in ulcerative colitis is poorly understood it has been suggested that the fibrinolytic system may play an important role. Evidence that local fibrinolytic activity does exist in the colon is accumulating and indicates that this may be enhanced in the active phase of the colitis (Kwaan, Cocco and Mendeloff, 1964; Kwaan et al., 1969). Against such a background, a trial of antifibrinolytic drugs in an attempt to control bleeding in proctocolitis is reasonable. Several such studies have been reported using the drug epsilon aminocaproic acid (EACA) and have yielded conflicting results. Salter and Read (1970) in an uncontrolled trial found EACA to be of value in 8 of 11 patients, whilst Mowat et al. (1973) using a double-blind cross-over technique found no significant reduction in faecal blood loss in their 13 cases.

An alternative antifibrinolytic agent in the form of tranexamic acid is available and although its mechanism of action is similar to that of EACA its potency is increased by a factor of at least 10. This drug is less likely to produce troublesome side effects such as nausea and vomiting.

The aim of the present study was to assess the value of tranexamic acid as a means of controlling bleeding in ulcerative proctocolitis.

\section{Patients and methods \\ Patients}

Informed consent was obtained from 15 patients for inclusion in this study. The diagnosis of ulcerative proctitis or proctosigmoiditis was made in all cases on the basis of clinical features, sigmoidoscopic appearances, barium enema examination and the histology of specimens of rectal mucosa.

All patients chosen for the study were in a stable phase of the disease but continued to see blood in most or all stools. Medication commenced before the trial started was continued unchanged. All patients were receiving oral iron supplements.

Details of patient age, sex, duration of disease, extent of disease, associated drug treatment and haemoglobin can be found in Table 1 for those patients included in the final analysis. 
TABLE 1. Clinical description of patients with ulcerative colitis in this study. All patients were on oral iron supplements

\begin{tabular}{|c|c|c|c|c|c|}
\hline Patient & Age (years) (Sex) & $\begin{array}{l}\text { Haemoglobin } \\
\qquad(\mathrm{g} / \mathrm{dl})\end{array}$ & $\begin{array}{l}\text { Duration of } \\
\text { disease (years) }\end{array}$ & Extent of disease & Other treatment \\
\hline 1 & 19(M) & $13 \cdot 0$ & 6 & Rectum & SASP $1 \mathrm{~g}$ thrice/day \\
\hline 2 & $30(\mathrm{~F})$ & $13 \cdot 4$ & 2 & Rectum & SASP $1 \mathrm{~g}$ thrice/day \\
\hline 3 & $65(F)$ & $11 \cdot 9$ & 15 & Rectum & SASP $1 \mathrm{~g}$ thrice/day \\
\hline 4 & $57(\mathrm{~F})$ & $12 \cdot 7$ & 4 & Recto-sigmoid & SASP $1 \mathrm{~g}$ thrice/day \\
\hline 5 & $40(F)$ & $13 \cdot 3$ & 10 & Rectum & Folic acid $5 \mathrm{mg}$ \\
\hline 6 & $38(F)$ & $11 \cdot 5$ & 5 & Rectum & $\begin{array}{l}\text { SASP } 1 \mathrm{~g} \text { thrice/day } \\
\text { Normacol }\end{array}$ \\
\hline 7 & $71(F)$ & $12 \cdot 8$ & 3 & Rectum & Normacol \\
\hline 8 & $20(M)$ & $11 \cdot 6$ & 5 & Recto-sigmoid & SASP 1 g thrice/day \\
\hline 9 & $47(M)$ & $13 \cdot 5$ & 2 & Recto-sigmoid & $\begin{array}{l}\text { SASP } 1 \mathrm{~g} \text { thrice/day } \\
\text { Pred. } 5 \mathrm{mg} \text { twice/day }\end{array}$ \\
\hline 10 & $26(F)$ & $12 \cdot 3$ & 1 & Rectum & $\begin{array}{l}\text { SASP } 1 \text { g thrice/day } \\
\text { Normacol }\end{array}$ \\
\hline 11 & $37(F)$ & $9 \cdot 7$ & 2 & Recto-sigmoid & SASP $1 \mathrm{~g}$ thrice/day \\
\hline 12 & $56(M)$ & $13 \cdot 2$ & 1 & Rectum & $\begin{array}{l}\text { SASP } 1 \mathrm{~g} \text { thrice/day } \\
\text { Ibuprofen } 200 \mathrm{mg} \text { thrice/day }\end{array}$ \\
\hline
\end{tabular}

SASP $=$ sulphasalazine

\section{Trial design}

The trial was designed to be a double-blind crossover study comparing tablets of tranexamic acid with an identical placebo. Patients were randomly allocated to receive a 3-week course of either tranexamic acid $1.5 \mathrm{~g}$ thrice daily or tablets of an identical placebo. At the end of the 3-week period they were crossed over for a further 3 weeks so that those previously receiving tranexamic acid received placebo and vice versa. Blood samples for haematological and biochemical profile were taken before drug treatment commenced and again on completion of the trial.

All patients were seen at weekly intervals for clinical assessment. Sigmoidoscopy was performed before entry to the trial and repeated at the end of each treatment period. Assessments and sigmoidoscopies were carried out by one observer only (D.H.).

\section{Grading scale}

The grading of sigmoidoscopic appearances was as follows: Grade 0 indicated a normal mucosa; Grade 1 implied mucosal erythema but the vessel pattern was retained; Grade 2 constituted mucosal oedema with absent vessel pattern; Grade 3 added contact bleeding with little or no blood or mucopus in the bowel lumen; and Grade 4 implied gross contact bleeding with spontaneous haemorrhage ahead of the sigmoidoscope.

Diary cards were issued and patients asked to provide a daily record of stool frequency, stool consistency, blood in stool and side effects of the treatment. Blood content of the stool was assessed as either present or absent and no attempt was made to estimate quantity.

\section{Fibrinolytic activity}

Fibrinolytic activity of venous blood samples $\stackrel{\mathbb{D}}{\mathrm{C}}$ taken from the antecubital fossa was measured using a 5-min venous occlusion test (Walker, Davidson and Hutton, 1976) and fibrin plate technique, $\overrightarrow{0}$ inoculated with resuspended euglobulin precipitas (Astrup and Mullertz, 1952; Kruft, 1976). This w高 done on 10 patients at the end of the placebo an active drug assessment periods.

\section{Results}

The results are presented in Table 2.

\section{Withdrawals}

Of the 15 patients originally included in the study there were 3 withdrawals. The reasons were an acute relapse necessitating the use of steroids (one case), a severe psychiatric upset (one case) and the revision of the diagnosis to Crohn's disease during the trial (one case).

\section{Blood in stool}

The effect on the outcome of this cross-over trial 음 resulting from the order in which the 2 treatments $\frac{D}{2}$ were administered was investigated (Hills and Armitage, 1979) but no time effect was found $\tilde{N}^{\circ}$ (Mann-Whitney U-test: $P>0.85$ ). The patients were therefore pooled into a single group and $\mathcal{O}_{0}$ treatment order ignored.

It was found that the proportion of stools containing visible blood was substantially lower in 60 patients (Nos $1,4,5,6,8,9)$ while taking tranexamic $₫$ acid. Two patients (Nos 3,7 ) bled slightly less on $\stackrel{?}{?}$ placebo, while for the remaining 4 patients there ${ }^{\circ}$ was no difference between the 2 treatments (Table 2). 
TABLE 2. Effects of tranexamic acid therapy on rectal appearance and bleeding. Patients marked with an asterisk* received tranexamic acid first. The blood in stool data are presented as stools with visible blood (upper number) over the total number of stools passed (lower number) in each treatment period

\begin{tabular}{ccccccc}
\hline & \multicolumn{3}{c}{ Sigmoidoscopic grading } & & \multicolumn{2}{c}{ Blood in stool } \\
\cline { 2 - 3 } \cline { 6 - 7 } Patient & Before trial & Tranexamic acid & Placebo & & Tranexamic acid & Placebo \\
\hline 1 & 2 & 0 & 1 & & $1 / 15$ & $7 / 23$ \\
2 & 3 & 1 & 0 & & $0 / 43$ & $0 / 40$ \\
3 & 4 & 4 & 4 & & $24 / 63$ & $20 / 66$ \\
4 & 4 & 2 & 4 & & $1 / 21$ & $8 / 38$ \\
5 & 4 & 2 & 2 & & $15 / 39$ & $35 / 38$ \\
$6^{*}$ & 2 & 1 & 3 & & $10 / 29$ & $37 / 43$ \\
$7^{*}$ & 3 & 1 & 1 & & $1 / 45$ & $0 / 31$ \\
$8^{*}$ & 3 & 2 & 3 & & $26 / 76$ & $66 / 71$ \\
9 & 3 & 2 & 2 & & $10 / 131$ & $27 / 158$ \\
$10^{*}$ & 3 & 4 & 3 & & $18 / 18$ & $16 / 16$ \\
$1^{*}$ & 4 & 3 & 4 & & $60 / 60$ & $63 / 63$ \\
$12^{*}$ & 4 & 3 & & $67 / 67$ & $63 / 63$ \\
\hline
\end{tabular}

Overall, the proportion of stools containing visible blood was significantly lower on tranexamic acid than on placebo (Wilcoxon matched pairs ranks sum test: $P<0.05)$.

\section{Sigmoidoscopic grading}

Statistically significant changes in the sigmoidoscopic grading of rectal mucosa were observed during the trial (Friendman two-way analysis of variance: $\left.\chi^{2}(2)=9 \cdot 188, P<0.01\right)$. Gradings were lower at the end of both treatment periods compared with the base-line grading before the trial although only the grading improvement during the tranexamic acid period was statistically significant $(P<0.05)$. No significant difference was demonstrated when appearances during the tranexamic acid period and those on placebo were compared.

\section{Frequency of defaecation and stool consistency}

No change occurred in either of these variables during treatment with tranexamic acid or placebo.

\section{Fibrinolytic activity}

In order to test the adequacy of the dose of tranexamic acid employed an assessment was made of fibrinolytic activity in peripheral venous blood. Using the paired $t$-test a significant difference was found in ten patients whose venous blood was sampled pre- and post-occlusion while on placebo $(P<0.05)$. The test was repeated on the same ten cases while on tranexamic acid and no significant difference was found in fibrinolytic activity between the pre- and post-occlusion venous samples.

\section{Side effects}

Nine patients reported no side effects. Two patients experienced some nausea (one with vomiting) which lasted from $24-48 \mathrm{hr}$ but in neither case did it require discontinuing treatment. One was on tranexamic acid and the other on placebo when symptoms occurred. One female patient volunteered the information that menstruation had been uncharacteristically light while taking tranexamic acid.

Routine haematology and biochemical profiles of venous blood samples taken at the beginning and close of the trial showed no abnormality which could be attributed to the medication.

\section{Patients' preference}

Patient preference for the trial medication at the close of the study was not routinely sought but of 3 patients volunteering a preference, all identified tranexamic acid as more beneficial.

\section{Discussion}

This work suggests that orally administered tranexamic acid can be of value for the control of rectal bleeding in some cases of ulcerative proctitis. The results accord well with the work of previous investigators (Salter and Read, 1970; Nilsson, Anderson and Bjorkman, 1966) who used epsilon aminocaproic acid as their antifibrinolytic agent. Unlike them, however, the present authors did not encounter the severe side effects of nausea, vomiting and diarrhoea which necessitated withdrawal of treatment in many instances and consequently they feel that tranexamic acid is superior in this respect.

Assessment of blood loss required the patient to record if visible blood was present or absent from each stool. This approach would not identify any reduction in bleeding short of complete cessation and therefore the actual diminution in blood loss on tranexamic acid could well be more impressive than the present results suggest. Further work involving the quantification of faecal blood loss would be 
needed to evaluate this. Such an approach may also explain why some patients, notably cases 10,11 and 12 (Table 2) apparently did not respond to the active drug. The figures do not rule out the possibility that these patients experienced a reduction in bleeding while on tranexamic acid but that this was not sufficient to eliminate all visible blood from the stool.

Activity of antifibrinolytic drug in reducing bleeding in ulcerative colitis raises some interesting questions as to its mechanism of action. The aetiology of ulcerative colitis is unknown but it has been shown that colonic tissue is able to exhibit fibrinolytic activity. Swan, Alexander-Williams and Cooke (1970) demonstrated that the activity of colonic arterial blood is enhanced as it passes through the colonic capillary bed and that this enhancement occurs in both inflammatory and non-inflammatory diseases of the colon. The presence of tissue and vascular activator of plasminogen in both normal and inflamed rectal biopsy specimens is well known and can be localized to crypt abcesses (Kwaan et al., 1964), lamina propria, and vascular endothelium (Kwaan et al., 1969). Such activity is greatly enhanced in active ulcerative colitis when compared to the quiescent phase of the disease. Demonstration of plasminogen activator in surface epithelial cells of cornea (Pandolfi and Astrup, 1967) and vagina (Tymparidis, King and Astrup, 1968) may also imply that similar activity resides in the epithelial cells of the large bowel.

Similar results have also been obtained in the case of stomach. They demonstrate an increase in fibrinolytic activity of gastric venous blood compared to gastric arterial blood and high concentrations of plasminogen activator in gastric mucosa. The implication for gastric haemorrhage and the value of EACA in this condition have been explored (Poller, 1979).

In the case of ulcerative colitis it could be suggested that a primary inflammatory reaction of unknown origin causes an increase in tissue activator concentrations and that disruption of cells allows this activity to be released. The resultant free plasmin then contributes to the initiation, enhancement and maintenance of the familiar bleeding of ulcerative colitis. Varying levels of fibrinolytic activity from patient to patient may help explain differing tendencies to bleed (even in the presence of continued diarrhoea) and also the variability of the response to antifibrinolytic drugs observed both in the present study and in previous work. This mechanism requires a local activation of fibrinolysis within colonic tissue only and is quite consistent with the fact that no general enhancement of fibrinolytic activity has been demonstrated in peripheral venous blood in ulcerative colitis (Salter and Read, 1970). In this context the use of tran $z$ examic acid in enema form would be interesting. $\stackrel{\AA}{\varrho}$ Overall, the authors' assessment of the appearance. $\subseteq$ of rectal mucosa at sigmoidoscopy accords well with $\vec{F}$ the reduction in mucosal haemorrhage with a signifi- $\frac{?}{5}$ cant improvement over the pre-trial grade occurring 응 when the patients were on tranexamic acid $(P<0.05) \cdot \frac{\bar{\sigma}}{\bar{c}}$.

As might be expected there was no significant $\frac{\rho}{\widetilde{\sigma}}$ difference between pre-trial and placebo gradings but $\stackrel{\mathbb{}}{\varrho}$ comparison of placebo and tranexamic acid gradings also revealed no significant difference. Exactly why $\overrightarrow{0}$ sigmoidoscopic appearances should have improved $\rightarrow$ at all on placebo is not clear but the improvement on $\vec{\omega}$ tranexamic acid was undoubtedly more impressive.

In conclusion, it is felt that oral tranexamic acido in a total dose of $4.5 \mathrm{~g}$ /day is of value in controlling $\frac{3}{6}$. mucosal haemorrhage in some patients with ulcer-o ative colitis and that this is achieved with minimum of side effects. It does not confer any benefit in terms of reducing the frequency of diarrhoea or $\stackrel{\infty}{-}$ improving the consistency of the stool. In the 은 present state of knowledge it is suggested that this drug may have a role to play alongside orthodox treatment in selected patients in whom bleeding is a $\underset{C}{C}$ prominent symptom.

\section{Acknowledgments}

We thank KabiVitrum Ltd for their financial support ate for providing tablets of tranexamic acid and placebo. Statistical analysis was carried out by Mr K. F. Yee a typing of the manuscript by Mrs Susan Davies. Nursing assistance was provided by Sister Morag Ravenscroft.

\section{References}

Astrup, T. \& Mullertz, S. (1952) The fibrin plate method for estimating fibrinolytic activity. Archives of Biochemistry and Biophysics, 40, 346.

Hills, M. \& ARMitage, P. (1979) The two period cross over clinical trial. British Journal of Clinical Pharmacology, 8, 7.

KLUFT, C. (1976) Occurrence of C1-inactivator and other proteinase inhibitors in euglobulin fraction and their 3 influence on fibrinolytic activity. Haemostasis, 5, 136.

KWaAN, H.C., Cocco, A. \& Mendeloff, A.I. (1964) Histologic demonstration of plasminogen activation in rectal biopsies from patients with active ulcerative colitis. $\bigcirc$ Journal of Laboratory and Clinical Medicine, 64, 877.

KWaAn, H.C., Cocco, A., Mendeloff, A.I. \& Astrup, T. (1969) Fibrinolytic activity in the normal and inflamed rectal mucosa. Scandinavian Journal of Gastroenterology, $4, \underset{D}{D}$ 441.

Mowat, N.A.G., Douglas, A.S., Brunt, P.W., McIntosh, J.A.R., KING, P.C. \& BodDy, K. (1973) Epsilon-aminocaproic acid therapy in ulcerative colitis. Digestive Diseases, $18,959$.

Nilsson, I.M., ANDERSON, L. \& BJorkmaN, S.E. (1966) Nు Epsilon-aminocaproic acid (EACA) as a therapeutic $\sigma$ agent based on five years' clinical experience. Acta medica scandinavica, 180 (Suppl. 448), 1.

ORMEROD, T.P. (1968) Anaemia in ulcerative colitis. Pro- $\overline{\mathscr{D}}$ ceedings of the Royal Society of Medicine, 61, 931.

Pandolfi, M. \& Astrup, T. (1967) A histochemical study of the fibrinolytic activity of cornea, conjunctiva and lachrymal gland. Archives of Ophthalmology, 77, 258. 
Poller, L. (1979) Fibrinolysis and gastrointestinal haemorrhage. Journal of Clinical Pathology, 33 (Suppl. (Roy. Coll. Path.) 14), 63.

SAlter, R.H. \& READ, A.E. (1970) Epsilon-aminocaproic acid therapy in ulcerative colitis. Gut, 11, 585.

Swan, C.H.J., AleXander-Williams, J. \& Cooke, W.T. (1970) Fibrinolysis in colonic diseases. Gut, 11, 588.
Tymparidis, K., King, A.E. \& Astrup, T. (1968) Fibrinolytically active cells in human vaginal smears. American Journal of Obstetrics and Gynecology, 100, 185.

WALKer, I.D., Davidson, J.F. \& HuTton, I. (1976) Fibrinolytic potential. The response to a five minute venous occlusion test. Thrombosis Research, 8, 629. 